\title{
Quasi-Chemical Approximation for Nonrandomness in the Hole Theory of Polymer Systems Comprising Random Copolymers
}

\author{
Hankun $\mathrm{XIE}^{\dagger}$ and Erik NIES* \\ Department of Basic Sciences, China Textile University, Shanghai, People's Republic of China \\ * Department of Polymer Technology, Eindhoven University of Technology, \\ P.O. Box 513, 5600MB, Eindhoven, The Netherlands
}

(Received May 25, 1998)

\begin{abstract}
A modified version of Simha-Somcynsky's hole theory developed earlier is adapted to obtain the equation of state and Helmholtz free energy for random copolymer systems and random copolymer/homopolymer blends. Miscibility behavior of the blend of poly(styrene-co-p-bromostyrene)/polystyrene is investigated to test the validity of the theory. With the molecular parameters extracted from the experimental PVT data, not only a satisfactory description of the experimental PVT surfaces is obtained, but also the upper critical miscibility behavior of the blend is predicted, which is in agreement with the experiment.
\end{abstract}

KEY WORDS Quasi-Chemical Approximation / Hole Theory / Random Copolymer /

It has been known for 30 years that equation of state properties determine the equilibrium thermodynamic properties of polymer systyms. This was first demonstrated by the cell model of Prigogine ${ }^{1}$ and later by the equation of state theory of Flory, Orwoll, and Vrij. ${ }^{2-4}$ The classical equation of state theories can be subdivided in three main classes ${ }^{5}$ and we have been particularly interested in the hole theory of Simha and Somcynsky (S-S theory) ${ }^{6}$. In the past few years, a modified version of the S-S theory, called Holey Huggins or $\mathrm{H}-\mathrm{H}$ theory, was established ${ }^{5,7}$ and then the $\mathrm{H}-\mathrm{H}$ theory was further modified to take into account nonrandomness of the system. ${ }^{8,9}$ While for most conventional polymer systems with van der waals type interactions, the assumption of random mixing is a good approximation, the effect of nonrandomness could be serious for systems with strong specific interactions, and the non-random $\mathrm{H}-\mathrm{H}$ theory paves the way for a satisfactory description of the latter systems. In this paper, the non-random $\mathrm{H}-\mathrm{H}$ theory is adapted to obtain the equation of state and Helmholtz free energy of random copolymers and random copolymer/homopolymer blends due to theoretical interest as well as practical importance. ${ }^{10}$ For simplicity, the theory presented is only for binary mixtures with one component being a random copolymer. However, the extention to more complicated systems is, in principle, straightforward. As an example of the application of the present theory, miscibility behavior of the blend of poly(styrene-co-p-bromostyrene)/polystyrene is investigated. With the molecular parameters extracted from the experimental PVT data of polystyrene, poly $(p$ bromostyrene) and a random copolymer poly(styreneco-p-bromostyrene) of known composition, not only a satisfactory description of the experimental PVT surfaces of the copolymer is obtained, but also the upper critical miscibility behavior of the blend is predicted, which is in qualitative agreement with the experiment.

\section{THEORY FOR RANDOM COPOLYMERS}

Consider a system of monodisperse random copolymer $A$ which comprises two different kinds of segments $A_{1}$ and $\mathrm{A}_{2}$ with a fraction of $\mathrm{A}_{1}$ segments being $p_{1}$ and total number of molecules being $N$. The following parameters characterize the system: $s$, the number of segments of each molecule; $3 c$, the number of external degrees of freedom of a molecule; $M_{0}$, the molar mass of the copolymer; $P, V$, and $T$, the pressure, volume and temperature of the system. In order to develop the theory, some other parameters are necessary, which are given below.

$z$ : the coordination number of the lattice

$N_{\mathrm{h}}$ : the number of holes in the lattice

$y$ : the occupied site fraction, $y=\frac{N s}{N s+N_{\mathrm{h}}}$

$\Phi_{1}\left(\Phi_{2}\right)$ : the segment fraction of species $\mathrm{A}_{1}\left(\mathrm{~A}_{2}\right)$

$M_{01}\left(M_{02}\right)$ : the molar mass of homopolymer $\mathrm{P}\left(\mathrm{A}_{1}\right)\left(\mathrm{P}\left(\mathrm{A}_{2}\right)\right)$ with the same number of segments $s$

$c_{\mathrm{s}}$ : the flexibility parameter per segment, $c_{\mathrm{s}}=c / s$

$c_{\mathrm{s} 1}\left(c_{\mathrm{s} 2}\right)$ : the flexibility parameter per segment of homopolymer $\mathrm{P}\left(\mathrm{A}_{1}\right)\left(\mathrm{P}\left(\mathrm{A}_{2}\right)\right)$

$z q_{\mathrm{c}}$ : the number of external contact sites of each molecule, $z q_{\mathrm{c}}=s(z-2)+2$

$u_{1}\left(u_{2}\right)$ : the contact site fraction of species $\mathrm{A}_{1}\left(\mathrm{~A}_{2}\right)$, $u_{1}=\frac{N z q_{\mathrm{c}} P_{1}}{N z q_{\mathrm{c}}+N_{\mathrm{h}} z}, \quad u_{2}=\frac{N z q_{\mathrm{c}}\left(1-p_{1}\right)}{N z q_{\mathrm{c}}+N_{\mathrm{h}} z}$

$q$ : the total contact site fraction of species $\mathrm{A}_{1}$ and $\mathrm{A}_{2}, q=u_{1}+u_{2}$

$v:$ the contact site fraction of holes, $v=1-q$

$Q$ : the total number of external contact pairs, $Q=z\left(N q_{\mathrm{c}}+N_{\mathrm{h}}\right) / 2$

$\tilde{\omega}: \quad$ reduced cell volume, $\tilde{\omega}=y \tilde{V}$ where $\tilde{V}=V /$ $\left\langle v^{*}\right\rangle$ is the reduced volume and $\left\langle v^{*}\right\rangle$ is the average hard core volume

$\beta$ : an auxiliary parameter, $\beta=2^{-1 / 6} \tilde{\omega}^{1 / 3}$

$\gamma:$ an auxiliary parameter, $\gamma=2 / z$

† To whom correspondence should be addressed. 
Table I. Enumeration of contacts in random copolymers

\begin{tabular}{|c|c|}
\hline Kind of contact pairs & Number of contact pairs \\
\hline $\begin{array}{l}\text { Segments } a_{1}-a_{2} \text { or } a_{2}-a_{1} \\
\text { Segments } a_{2}-\text { Hole or Hole- } a_{2} \\
\text { Segments } a_{1}-\text { Hole or Hole- } a_{1} \\
\text { Segments } a_{1}-a_{1} \\
\text { Segments } a_{2} a_{2} \\
\text { Hole-hole }\end{array}$ & $\begin{array}{l}\quad Q x_{1} \\
Q x_{2} \\
Q x_{3} \\
Q\left(u_{1}-x_{1}-x_{3}\right) \\
Q\left(v-x_{2}-x_{3}\right)\end{array}$ \\
\hline
\end{tabular}

$\alpha:$ an auxiliary parameter, $\alpha=\gamma(1-1 / s)$

$n$ : the number of moles of the system

For a system of random copolymers containing two kinds of segments, there are six different kinds of contacts and three extra parameters $x_{1}, x_{2}$, and $x_{3}$ are necessary to enumerate the number of different contacts as shown in Table I, which is the same as the situation occurring in binary mixtures ( $c f$. Table I of ref 9 ).

The partition function of the system is given by

$$
Z\left(N, p_{1}, N_{\mathrm{h}}, V, T\right)=\sum_{x_{1}, x_{2}, x_{3}} Z_{x}\left(N, p_{1}, N_{\mathrm{h}}, V, T, x_{1}, x_{2}, x_{3}\right)
$$

where the partition function of the subsystem $Z_{x}$ has the form

$$
Z_{X}=g l_{\mathrm{f}}^{3 c_{\mathrm{s}} N s} \exp \left(-E_{0} / k T\right)
$$

The combinatorial factor $g$ in eq 2 is similar to the same factor for binary mixtures ( $c f$ eq A3 or ref 9):

$$
g=N_{\mathrm{hh}} \frac{\left[Q\left(u_{1}-x_{1}^{*}-x_{3}^{*}\right)\right] !\left[Q\left(u_{2}-x_{1}^{*}-x_{2}^{*}\right)\right] !\left[Q\left(v-x_{2}^{*}-x_{3}^{*}\right)\right] !\left[\left(Q x_{1}^{*}\right) !\right]^{2}\left[\left(Q x_{2}^{*}\right) !\left[\left(Q x_{3}^{*}\right) !\right]^{2}\right.}{\left[Q\left(u_{1}-x_{1}-x_{3}\right)\right] !\left[Q\left(u_{2}-x_{1}-x_{2}\right)\right] !\left[Q\left(v-x_{2}-x_{3}\right)\right] !\left[\left(Q x_{1}\right) !\right]^{2}\left[\left(Q x_{2}\right) !\right]^{2}\left[\left(Q x_{3}\right) !\right]^{2}}
$$

where $x_{1}^{*}=u_{1} u_{2}, x_{2}^{*}=u_{2} v$ and $x_{3}^{*}=u_{1} v$.

The only difference lies in the slightly different expression for $N_{\mathrm{hh}}$, since now we have

$$
\begin{aligned}
\ln N_{\mathrm{hh}}= & N s\left[-\frac{1}{s} \ln y-\frac{1-y}{y} \ln (1-y)\right. \\
& \left.+\frac{1}{\gamma}\left(\frac{1-\alpha y}{y}\right) \ln (1-\alpha y)\right]
\end{aligned}
$$

If one compares eq 4 to the corresponding equation for the binary mixture, one finds that only a term $N\langle s\rangle\left(\varphi_{\mathbf{A}} \ln \varphi_{\mathrm{A}} / s_{\mathrm{A}}+\varphi_{\mathbf{B}} \ln \varphi_{\mathbf{B}} / s_{\mathbf{B}}\right)$, which accounts for the entropy of mixing, is missing. The expression for segmental free length $l_{\mathrm{f}}$ of the copolymer is exactly the same as that for binary mixtures ( $c f$. eq A4 in ref 9), and the internal energy $E_{0}$ is also the same if one replaces $\mathrm{s}$ by $\langle s\rangle$. So, the detailed forms will not be given here. The obvious reason for this identity is that there are two different kinds of segments in the copolymer just as in the mixture.

The maximization of the partition function $Z_{x}$ leads to a set of equations for the equilibrium values of $x_{i}$ denoted by $\bar{x}_{i}(i=1,2,3)$, which are exactly the same as that for a binary mixture (see eq A 19 of ref 9) and the final expressions for the configurational partition function and Helmholtz free energy are also similar to the mixture. For the sake of convenience, we present here only the equation for the free energy, which reads

$$
\begin{aligned}
A / n s R T= & \frac{1}{s} \ln y+\frac{1-y}{y} \ln (1-y)-\frac{1-\alpha y}{\gamma y} \ln (1-\alpha y) \\
& -\frac{z(1-\alpha)}{2 q}\left[2 u_{1} \ln u_{1}+2 u_{2} \ln u_{2}\right. \\
& +2 v \ln v-\left(u_{1}-\bar{x}_{1}-\bar{x}_{3}\right) \ln \left(u_{1}-\bar{x}_{1}-\bar{x}_{3}\right) \\
& -\left(u_{2}-\bar{x}_{1}-\bar{x}_{2}\right) \ln \left(u_{2}-\bar{x}_{1}-\bar{x}_{2}\right) \\
& -\left(v-\bar{x}_{2}-\bar{x}_{3}\right) \ln \left(v-\bar{x}_{2}-\bar{x}_{3}\right)-2 \bar{x}_{1} \ln \bar{x}_{1} \\
& \left.-2 \bar{x}_{2} \ln \bar{x}_{2}-2 \bar{x}_{3} \ln \bar{x}_{3}\right]
\end{aligned}
$$

$$
\begin{aligned}
& -c_{3} \ln \left\{\left\langle v^{*}\right\rangle \tilde{\omega}\left[1-\beta\left(1-\frac{\bar{x}}{q}\right)\right]^{3}\right\} \\
& +\frac{c_{\mathrm{s}}}{2 \tilde{T}}\left(1-\frac{\bar{x}}{q}\right)\left(A \tilde{\omega}^{-4}-2 B \tilde{\omega}^{-2}\right) \\
& +k_{1} c_{\mathrm{s}} \ln \left(k_{2} M_{0} T\right)
\end{aligned}
$$

where $\bar{x}=\bar{x}_{2}+\bar{x}_{3}$ and the term $k_{1} c_{\mathrm{s}} \ln \left(k_{2} M_{0} T\right)$ comes from the kinetic part of the partition function.

The equation of state of the copolymer is given by

$$
P=-\left.\frac{\partial A}{\partial V}\right|_{N, T, y, P_{1}}
$$

which also has the same form as that for binary mixtures if one replaces $s$ by $\langle s\rangle$ and correspondingly $\alpha$ by $\langle\alpha\rangle$ and $c_{\mathrm{s}}$ by $\left\langle c_{\mathrm{s}}\right\rangle$ :

$$
\begin{aligned}
\frac{\tilde{P} \tilde{V}}{\tilde{T}}= & \frac{1}{1-\beta\left(1-\frac{\tilde{x}}{q}\right)}+\frac{2}{\tilde{T}}\left(1-\frac{\tilde{x}}{q}\right)\left(A \tilde{\omega}^{-4}-B \tilde{\omega}^{-2}\right) \\
& +\left(m_{21}+m_{31}+\frac{m_{41}}{\tilde{T}}\right) \tilde{V} \frac{\partial \bar{x}_{1}}{\partial \tilde{V}} \\
& +\left(m_{22}+m_{31}+\frac{m_{42}}{\tilde{T}}\right) \tilde{V} \frac{\partial \bar{x}_{2}}{\partial \tilde{V}} \\
& +\left(m_{23}+m_{33}+\frac{m_{43}}{\tilde{T}}\right) \tilde{V} \frac{\partial \bar{x}_{3}}{\partial \tilde{V}}
\end{aligned}
$$

where

$$
\begin{aligned}
& m_{21}=-\frac{z(1-\langle\alpha\rangle)}{2 q\left\langle c_{\mathrm{s}}\right\rangle} \ln \left[\frac{\left(\overline{x_{1}}\right)^{2}}{\left(u_{1}-\overline{x_{1}}-\overline{x_{3}}\right)\left(u_{2}-\overline{x_{1}}-\overline{x_{2}}\right)}\right] \\
& m_{22}=-\frac{z(1-\langle\alpha\rangle)}{2 q\left\langle c_{\mathrm{s}}\right\rangle} \ln \left[\frac{\left(\overline{x_{2}}\right)^{2}}{\left(u_{2}-\overline{x_{1}}-\overline{x_{2}}\right)\left(v-\overline{x_{2}}-\overline{x_{3}}\right)}\right] \\
& m_{23}=-\frac{z(1-\langle\alpha\rangle)}{2 q\left\langle c_{\mathrm{s}}\right\rangle} \ln \left[\frac{\left(\overline{x_{3}}\right)^{2}}{\left(u_{1}-\overline{x_{1}}-\overline{x_{3}}\right)\left(v-\overline{x_{2}}-\overline{x_{3}}\right)}\right]
\end{aligned}
$$




$$
\begin{aligned}
m_{31}= & -\frac{1}{1-\beta\left(1-\frac{\bar{x}}{q}\right)}\left[\beta\left(1-\frac{\bar{x}}{q}\right) \frac{\partial \ln \left\langle v^{*}\right\rangle}{\partial \overline{x_{1}}}\right] \\
m_{32}= & \frac{1}{1-\beta\left(1-\frac{\bar{x}}{q}\right)}\left[\frac{3 \beta}{q}-\beta\left(1-\frac{\bar{x}}{q}\right) \frac{\partial\left\langle v^{*}\right\rangle}{\partial \bar{x}_{2}}\right] \\
m_{33}= & \frac{1}{1-\beta\left(1-\frac{\bar{x}}{q}\right)}\left[\frac{3 \beta}{q}-\beta\left(1-\frac{\bar{x}}{q}\right) \frac{\partial\left\langle v^{*}\right\rangle}{\partial \bar{x}_{3}}\right] \\
m_{41}= & -\frac{1}{2}\left(1-\frac{\bar{x}}{q}\right)\left[\left(A \tilde{\omega}^{-4}-2 B \tilde{\omega}^{-2}\right) \frac{\partial \ln \left\langle\varepsilon^{*}\right\rangle}{\partial \bar{x}_{1}}\right. \\
& \left.+4\left(A \tilde{\omega}^{-4}-B \tilde{\omega}^{-2}\right) \frac{\partial \ln \left\langle v^{*}\right\rangle}{\partial \bar{x}_{1}}\right] \\
m_{42}= & -\frac{1}{2}\left[\left(1-\frac{\bar{x}}{q}\right) \frac{\partial \ln \left\langle\varepsilon^{*}\right\rangle}{\partial \bar{x}_{2}}-\frac{1}{q}\right]\left(A \tilde{\omega}^{-4}-2 B \tilde{\omega}^{-2}\right) \\
& -2\left(1-\frac{\bar{x}}{q}\right)\left(A \tilde{\omega}^{-4}-B \tilde{\omega}^{-2}\right) \frac{\partial \ln \left\langle v^{*}\right\rangle}{\partial \bar{x}_{2}} \\
m_{43}= & -\frac{1}{2}\left[\left(1-\frac{\bar{x}}{q}\right) \frac{\partial \ln \left\langle\varepsilon^{*}\right\rangle}{\partial \bar{x}_{3}}-\frac{1}{q}\right]\left(A \tilde{\omega}^{-4}-2 B \tilde{\omega}^{-2}\right) \\
& -2\left(1-\frac{\bar{x}}{q}\right)\left(A \tilde{\omega}^{-4}-B \tilde{\omega}^{-2}\right) \frac{\partial \ln \left\langle v^{*}\right\rangle}{\partial \bar{x}_{3}}
\end{aligned}
$$

From the above derivation, it is obvious that from the theoretical point of view, a random copolymer is quite similar to a binary mixture rather than a single component polymer. This is manifested not only by the final result for the Helmholtz free energy and, especially, the equation of state, but also by the averages of the molecular parameters such as average hard core volume $\left\langle v^{*}\right\rangle$, average interaction parameter $\left\langle\varepsilon^{*}\right\rangle$ and segmental flexibility parameters $c_{\mathrm{s}} \cdot\left\langle v^{*}\right\rangle$ and $\left\langle\varepsilon^{*}\right\rangle$ are determined by the mixing rules:

$$
\begin{aligned}
& \left(u_{1}-\overline{x_{1}}-\overline{x_{3}}\right) \varepsilon_{\mathrm{A}_{1} \mathrm{~A}_{1}}^{*} v_{\mathrm{A}_{1} \mathrm{~A}_{1}}^{* 4}+\left(u_{2}-\overline{x_{1}}-\overline{x_{2}}\right) \varepsilon_{\mathrm{A}_{2} \mathrm{~A}_{2}}^{* 4} \\
& +2 \overline{x_{1}} \varepsilon_{\mathrm{A}_{1} \mathrm{~A}_{2}}^{*} v_{\mathrm{A}_{1} \mathrm{~A}_{2}}^{* 4}=(q-\bar{x})\left\langle\varepsilon^{*}\right\rangle\left\langle v^{*}\right\rangle^{4} \\
& \left(u_{1}-\bar{x}_{1}-\bar{x}_{3}\right) \varepsilon_{\mathrm{A}_{1} \mathrm{~A}_{1}}^{*} v_{\mathrm{A}_{1} \mathrm{~A}_{1}}^{* 2}+\left(u_{2}-\bar{x}_{1}-\bar{x}_{2}\right) \varepsilon_{\mathrm{A}_{2} \mathrm{~A}_{2}}^{* 2} \\
& \quad+2 \bar{x}_{1} \varepsilon_{\mathrm{A}_{1} \mathrm{~A}_{2}}^{*} v_{\mathrm{A}_{1} \mathrm{~A}_{2}}^{* 2}=(q-\bar{x})\left\langle\varepsilon^{*}\right\rangle\left\langle v^{*}\right\rangle^{2}
\end{aligned}
$$

where

$$
\begin{gathered}
\varepsilon_{\mathrm{A}_{1} \mathrm{~A}_{2}}^{*}=\delta e_{\mathrm{s}}\left(\varepsilon_{\mathrm{A}_{1} \mathrm{~A}_{1}}^{*} \varepsilon_{\mathrm{A}_{2} \mathrm{~A}_{2}}^{*}\right)^{1 / 2} \\
v_{\mathrm{A}_{1} \mathrm{~A}_{2}}^{*}=\delta v_{\mathrm{s}}\left[\left(v_{\mathrm{A}_{1} \mathrm{~A}_{1}}^{*}{ }^{1 / 3}+v_{\mathrm{A}_{2} \mathrm{~A}_{2}}^{*}{ }^{1 / 3}\right) / 2\right]^{3}
\end{gathered}
$$

and $c_{\mathrm{s}}$ is also an average over $c_{\mathrm{s} 1}$ and $c_{\mathrm{s} 2}$ defined as

$$
c_{\mathrm{s}}=c_{\mathrm{s} 1} \varphi_{1}+c_{\mathrm{s} 2} \varphi_{2}+\left(\delta c_{\mathrm{s}}-1\right) \sqrt{c_{\mathrm{s} 1} c_{\mathrm{s} 2}} \varphi_{1} \varphi_{2}
$$

Note that eq 11 is different from the average flexibility parameter of a binary mixture and the adjustable parameter $\delta c_{\mathrm{s}}$, which can be determined from the experimental PVT data of the copolymer, describes the deviation of the flexibility parameter of a random copolymer from that of a mixture. The introduction of $\delta c_{\mathrm{s}}$ seems crucial for the theory to obtain a very good description of the experimental PVT surfaces, since in an earlier publication, where $\delta c_{\mathrm{s}}$ was virtually set to be zero, there appeared some discrepancies between theory and experiment. ${ }^{11}$

\section{THEORY FOR RANDOM COPOLYMER/HOMOPOLYMER BLENDS}

The theory for pure random copolymer developed so far is readily extended to more complicated systems of random copolymer/homopolymer blends. Consider a monodisperse polymer system which comprises homopolymer $\mathrm{B}$ and random copolymer $\mathrm{A}$ with repeat units $A_{1}$ and $A_{2}$ and the mole fraction of $A_{1}$ segments in the copolymer is $p_{1}$. In the following, the copolymer as a whole will be treated as one of the two components and the strategy used in developing the theory for binary mixture can simply be used here as well, though the present situation is a little bit more complicated since we have more contact pairs to consider. The derivation is quite long and cumbersome, but is rather similar to that for binary homopolymer mixtures. In order not to bore the reader, we will omit the details of the derivation and only present the main result of the theory, i.e., the Helmholtz free energy of the system. The equation of state can be found in the APPENDIX.

According to the derivation, the Helmholtz free energy of random copolymer/homopolymer blends reads

$$
\begin{aligned}
& A / n\langle s\rangle R T=\varphi_{\mathrm{A}} \ln \varphi_{\mathrm{A}}+\varphi_{\mathrm{B}} \ln \varphi_{\mathrm{B}} \\
& +\frac{1}{\langle s\rangle} \ln y+\frac{1-y}{y} \ln (1-y)-\frac{1-\langle\alpha\rangle y}{\gamma y} \ln (1-\langle\alpha\rangle y) \\
& -\frac{z(1-\langle\alpha\rangle)}{2 q}\left[2 u_{1} \ln u_{1}+2 u_{2} \ln u_{2}+2 v \ln v\right. \\
& +2 w \ln w-\left(u_{1}-\bar{x}_{1}-\bar{x}_{2}-\bar{x}_{3}\right) \ln \left(u_{1}-\bar{x}_{1}-\bar{x}_{2}-\bar{x}_{3}\right) \\
& -\left(u_{2}-\bar{x}_{1}-\bar{x}_{4}-\bar{x}_{5}\right) \ln \left(u_{2}-\bar{x}_{1}-\bar{x}_{4}-\bar{x}_{5}\right) \\
& -\left(v-\bar{x}_{2}-x_{4}-\bar{x}_{6}\right) \ln \left(v-\bar{x}_{2}-\bar{x}_{4}-\bar{x}_{6}\right) \\
& \left.-\left(w-x_{3}-x_{5}-x_{6}\right) \ln \left(w-x_{3}-x_{5}-x_{6}\right)-2 \sum_{i=1}^{6} \bar{x}_{i} \ln \bar{x}_{i}\right] \\
& -\left\langle c_{\mathrm{s}}\right\rangle \ln \left\{\left\langle v^{*}\right\rangle \tilde{\omega}\left[1-\beta\left(1-\frac{\bar{x}}{q}\right)\right]^{3}\right\} \\
& +\frac{\left\langle c_{\mathrm{s}}\right\rangle}{2 \tilde{T}}\left(1-\frac{\bar{x}}{q}\right)\left(A \tilde{\omega}^{-4}-2 B \tilde{\omega}^{-2}\right)
\end{aligned}
$$

where $\bar{x}_{i}(i=1,6)$ are molecular parameters used in enumeration of contacts (see Table II), which characterize the extent of deviation from randomness and are determined by the quasi-chemical equations (see ref 9). The physical meaning of the other symbols is explained in APPENDIX A.

The first five terms on the r.h.s in eq 12 represent the combinatorial entropy of random-mixing of holes with homopolymer and copolymer segments. The terms in the square bracket account for the extra contribution due to non-random-mixing of holes and the different kinds of segments. The remaining terms stem from the contribution of free volume and internal energy. Notice that in the above equation, the kinetic term has been omitted.

The flexibility parameter $\left\langle c_{\mathrm{s}}\right\rangle$ in eq 12 is defined by 
Table II. Enumeration of contacts in random copolymer/homopolymer blends

\begin{tabular}{|c|c|}
\hline Kind of contact pairs & Number of contact pairs \\
\hline $\begin{array}{l}\text { Segments } a_{1}-a_{2} \text { or Segments } a_{2}-a_{1} \\
\text { Segments } a_{1}-b \text { or Segments } b-a_{1} \\
\text { Segment } a_{1}-\text { Hole or Hole-segment } a_{1} \\
\text { Segments } a_{2}-b \text { or Segments } b-a_{2} \\
\text { Segment } a_{2}-\text { Hole or Hole-Segment } a_{2} \\
\text { Segment } b-\text { Hole or Hole-Segment } b \\
\text { Segments } a_{1}-a_{1} \\
\text { Segments } a_{2}-a_{2} \\
\text { Segments } b-b \\
\text { Hole-hole }\end{array}$ & $\begin{array}{lr} & Q x_{1} \\
& Q x_{2} \\
Q x_{3} & Q x_{4} \\
& \\
Q x_{5} & \\
Q x_{6} & \\
Q\left(u_{1}-x_{1}-x_{2}-x_{3}\right) \\
Q\left(u_{2}-x_{1}-x_{4}-x_{5}\right) \\
Q\left(v-x_{2}-x_{4}-x_{6}\right) \\
Q\left(w-x_{3}-x_{5}-x_{6}\right)\end{array}$ \\
\hline
\end{tabular}

Table III. Molecular parameters for $\mathrm{pS}$ and $\mathrm{p}(\mathrm{pBrS})$

\begin{tabular}{|c|c|c|c|}
\hline & $\varepsilon_{i i}^{*}$ & $v_{i i}^{*}$ & \\
\hline & $10^{4} \mathrm{~J} \mathrm{~mol}^{-1}$ & $10^{-4} \mathrm{~m}^{3} \mathrm{~mol}^{-1}$ & \\
\hline $\mathrm{pS}$ & 0.8846 & 0.9525 & 1.3198 \\
\hline $\mathrm{p}(\mathrm{pBrS})$ & 1.2293 & 1.1497 & 1.8394 \\
\hline
\end{tabular}

Table IV. Cross parameters obtained from experimental PVT data of $\mathrm{p}(\mathrm{S}-\mathrm{co}-\mathrm{pBrS}) / \mathrm{pS}$ with a known composition

\begin{tabular}{ccc}
\hline$\delta \varepsilon_{\mathrm{S}}$ & $\delta v_{\mathrm{s}}$ & $\delta c_{\mathrm{s}}$ \\
\hline 1.0251 & 1.0230 & 0.7684 \\
\hline
\end{tabular}

$\left\langle c_{\mathrm{s}}\right\rangle=$

$\left[c_{\mathrm{sA}_{1}} \varphi_{\mathrm{A}_{1}}+c_{\mathrm{sA}_{2}} \varphi_{\mathrm{A}_{2}}+\left(\delta c_{\mathrm{s}}-1\right) \sqrt{c_{\mathrm{sA}_{1}} c_{\mathrm{sA}_{2}}} \varphi_{\mathrm{A}_{1}} \varphi_{\mathrm{A}_{2}}\right] \varphi_{\mathrm{A}}+c_{\mathrm{sB}} \varphi_{\mathrm{B}}$

where $c_{\mathrm{SA}_{1}}\left(c_{\mathrm{SA}_{2}}\right)$ is the flexibility parameter of the respective homopolymer $\mathrm{p}\left(\mathrm{A}_{1}\right)\left(\mathrm{p}\left(\mathrm{A}_{2}\right)\right) ; \varphi_{\mathrm{A}_{1}}\left(\varphi_{\mathrm{A}_{2}}\right)$ is the segment fraction of $\mathrm{A}_{1}\left(\mathrm{~A}_{2}\right)$ in the pure copolymer. Equation 10 once again expresses the assumption that a copolymer comprising segments $A_{1}$ and $A_{2}$ is characterized by a flexibility parameter different from that of a physical mixture of homopolymers $\mathrm{p}\left(\mathrm{A}_{1}\right)$ and $\mathrm{p}\left(\mathrm{A}_{2}\right)$. This is reflected in the non-unity value of the copolymer parameter $\delta c_{\mathrm{s}}$ (see Table IV).

\section{UCST MISCIBILITY BEHAVIOR OF THE BLEND POLY(STYRENE-co-p- BROMOSTYRENE)/POLYSTYRENE}

As an example of the application of the present theory, we have investigated the miscibility behavior of the blend poly(styrene-co- $p$-bromostyrene)/polystyrene(p(S-co$\mathrm{pBrS} / \mathrm{pS})$. Table III shows the molecular parameters for $\operatorname{pS}\left(\varepsilon_{\mathrm{A}_{1} \mathrm{~A}_{1}}^{*}, v_{\mathrm{A}_{1} \mathrm{~A}_{1}}^{*}, c_{\mathrm{sA}_{1}}\right)$ and $\mathrm{p}(\mathrm{pBrS})\left(\varepsilon_{\mathrm{A}_{2} \mathrm{~A}_{2}}^{*}, v_{\mathrm{A}_{2} \mathrm{~A}_{2}}^{*}, c_{\mathrm{sA}_{2}}\right)$ obtained from the experimental PVT data of the homopolymers with a multi-parameters-fitting program developed in Eindhoven University of Technology, which can solve the equation of state and the quasi-chemical equations simultaneously. The cross parameters $\varepsilon_{\mathbf{A}_{1} \mathrm{~A}_{2}}^{*}$, $v_{\mathrm{A}_{1} \mathrm{~A}_{2}}^{*}, \delta c_{\mathrm{s}}$, or equivalently $\delta e_{\mathrm{s}}, \delta v_{\mathrm{s}}, \delta c_{\mathrm{s}}$ are determined from the experimental PVT data of a copolymer with known composition $\mathrm{p}(\mathrm{S}(0.84)$-co-pBrS(0.16)) in a similar way. The cross parameter values obtained in such a
Spinodal PS/(P(S-co-pBrS) NRM Hole Theory

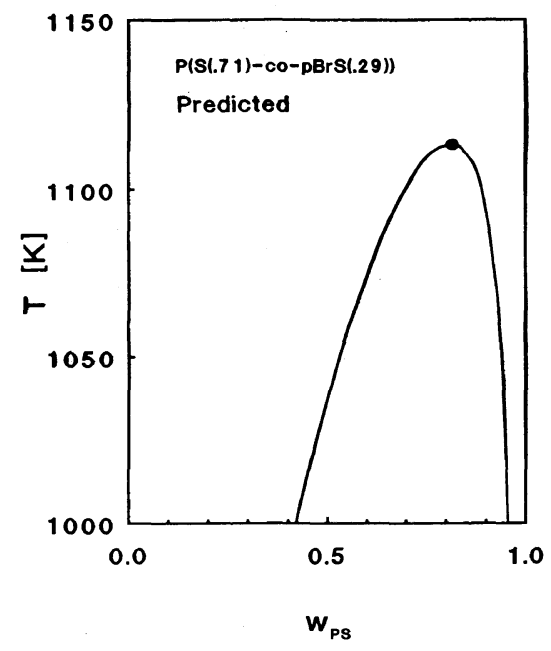

(a)

Spinodal PS/(P(S-co-pBrS) NRM Hole Theory

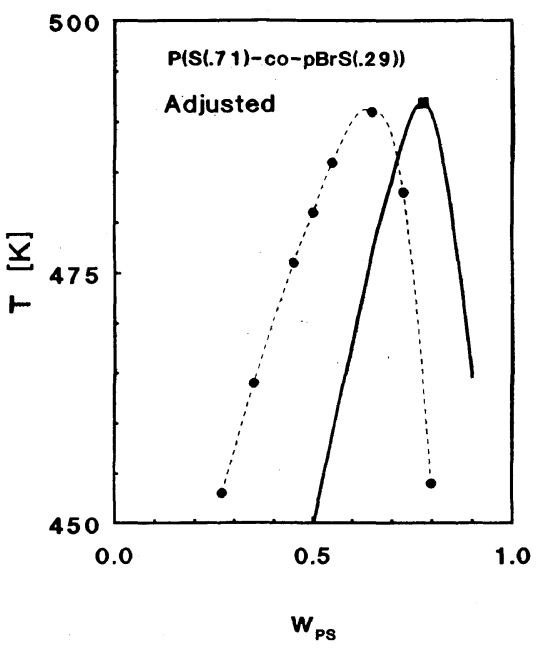

(b)

Figure 1. Miscibility behavior for the system $\mathrm{p}(\mathrm{S}(.71) c o-\mathrm{pBrS}(.29))$ / pS. (a) Predicted; (b) Adjusted; $(\cdots \bullet \cdots)$ : experimental data (ref 12); (—): computed.

way are shown in Table IV. It is interesting to see that the flexibility parameter of the random copolymer is smaller than that of a physical mixture of corresponding homopolymers, since $\delta c_{\mathrm{s}}$ assumes a value smaller than 1 , which implies that copolymerization makes the polymer chains less flexible.

With the parameters so far obtained, it is possible to predict miscibility behavior of the blend $\mathrm{p}(\mathrm{S}-\mathrm{co}$ $\mathrm{pBrS}) / \mathrm{pS}$ by the present theory, since now we have the fully determined Helmholtz free energy and hence Gibbs free energy of the mixture $(G=F+P V)$. The spinodal curve is then determined by the condition

$$
\left(\frac{\partial^{2} G}{\partial \varphi_{\mathrm{B}}^{2}}\right)_{P, T}=0
$$

Figure la shows the predicted UCST spinodal curve for the blend $\mathrm{p}(\mathrm{S}(0.71)-c o-\mathrm{pBrS}(0.29)) / \mathrm{pS}$. Although the predicted critical temperature is too high as compared to the experimental maximum temperature (below 
EoS $\mathrm{P}(\mathrm{S}-\mathrm{co}-\mathrm{pBrS})$

NRM Hole Theory

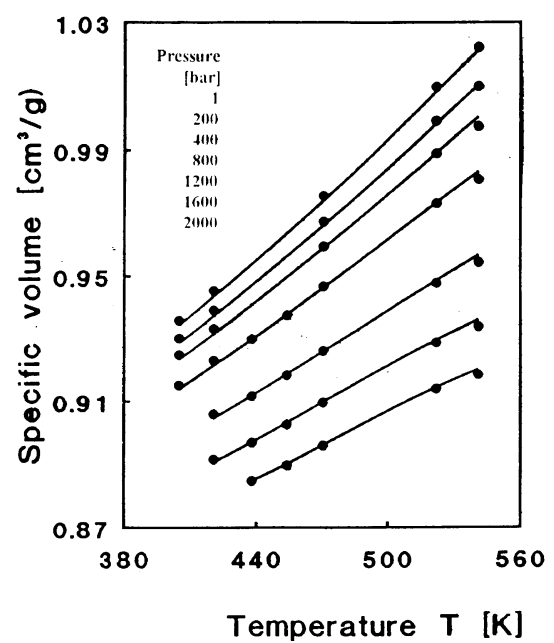

(a)

\section{EoS P(S-co-pBrS)} NRM Hole Theory

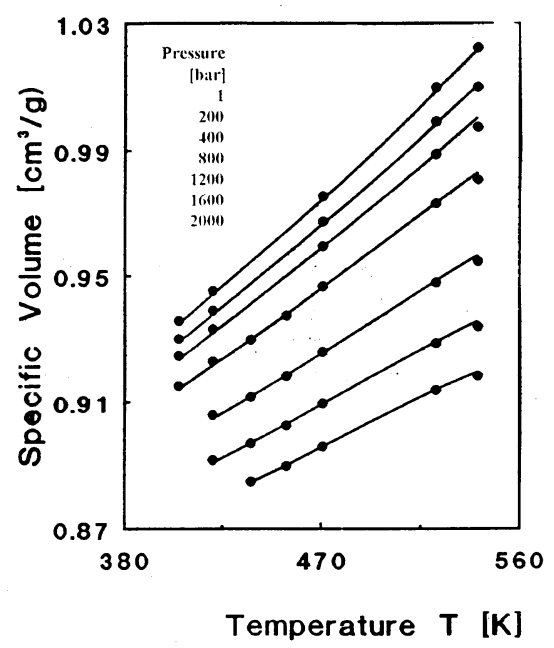

(b)

Figure 2. Equation of state behavior of the random copolymer $\mathrm{p}(\mathrm{S}(.84)-c o-\mathrm{pBrS}(.16))$. (a) Description; (b) Prediction; •: experimental data (ref 13); ( - ): computed.

$500 \mathrm{~K}$ ), the present theory does predict successfully the occurrence of UCST phase behavior of the system. It seems that the cross parameters obtained from PVT measurement of the random copolymer is not accurate enough for predicting miscibility behavior of the binary mixture of random copolymer and corresponding homopolymer quantitatively. However if allowance is made for small changes in the cross parameters $\delta e_{\mathrm{s}}$ and $\delta v_{\mathrm{s}}$, the experimental UCST gap can be described much more accurately whereas the PVT description of the copolymer can still be good enough. For instance, if we change $\delta e_{\mathrm{s}}$ from 1.0251 to 1.0201 and $\delta v_{\mathrm{s}}$ from 1.023 to 0.9872 respectively and keep $\delta c_{\mathrm{s}}$ unchanged, then, the corresponding spinodal curve is much closer to the experimental one (Figure 1b). Meanwhile, with these "adjusted" cross parameters we can still obtain very good PVT description of the copolymer as shown in Figure $2 \mathrm{~b}$. This is manifested by the fact that if one compares
Figure 2b with Figure 2a, which shows calculated PVT curves with cross parameters abstracted from the best fit of PVT data of the copolymer, no noticeable distinctions can be found.

It is worth mentioning also that for the present system, the effect of non-random-mixing is not crucial either for the PVT description of the copolymer or for predicting the UCST miscibility behavior of the mixture. In fact, if we replace the values of $\bar{x}_{i}$ 's in the Helmholtz free energy by $x_{i}^{*}$ 's, which are the values of $\bar{x}_{i}$ 's in the case of random mixing, we may recover the original random-mixing theory and the result shows that the description of PVT data as well as the prediction of miscibility behavior remain almost unchanged. The reason may be that there are no strong polar groups in the present system and nonrandomness is then not serious. However, due to the limited experimental PVT and miscibility data available, for the moment the present system is the only one suitable for the present investigation. Nevertheless, it may be expected that for polar polymer systems the non-randommixing theory presented in this paper will be able to offer better understanding than the original random-mixing theory.

\section{CONCLUSIONS}

The non-random-mixing version of Simha-Somcynsky's hole theory for disordered fluid state has been further extended to random copolymer systems and random copolymer/homopolymer mixtures. With the molecular parameters extracted from the experimental PVT data of the polymers, not only a satisfactory description of the experimental PVT surfaces can be obtained, it is even possible to predict the miscibility behavior of some random copolymer/homopolymer blends such as poly(styrene-co-p-bromostyrene)/polystyrene. Though the predicted critical temperature for $\mathrm{p}(\mathrm{S}-c o-p-\mathrm{BrS}) / \mathrm{pS}$ belnds is too high, the UCST phase behavior is actually predicted from the PVT measurement of the polymers. A much more precise description of the UCST phase behavior is realized if allowance is made for small changes in the cross parameters $\delta e_{\mathrm{s}}$ and $\delta v_{\mathrm{s}}$ and the PVT behavior of the random copolymer predicted with these adjusted cross parameters is in quantitative agreement with the experimental data. It seems that molecular parameters obtained from equation of state data are not sufficiently accurate to predict miscibility behavior quantitatively, though the basic phase behavior can be predicted correctly. In principle, the theory can be readily extended to randomcopolymer/copolymer blends. This is left for further exploration. ${ }^{1}$

Acknowledgment. The authors are indebted to professor Simha for stimulating discussions and critical reading of the manuscript. $\mathrm{H}$. Xie also wishes to express his sincere thanks to professor Simha for his hospitality.

\section{REFERENCES}

1. I. Prigogine, A. Bellemas, and V. Mathot, "The Molecular Theory of Solutions," North Holland Publishing Co., Amsterdam, 1957.

2. P. J. Flory, R. A. Orwoll, and A. Vrij, J. Am. Chem. Soc., 86 3567 (1964) 
3. P. J. Flory, J. Am. Chem. Soc., 87, 1833 (1965).

4. P. J. Flory, Discuss. Faraday Soc., 49, 7 (1970).

5. E. Nies and A. Strokes, Macromolecules, 23, 4088 (1990).

6. R. Simha and T. Somcynsky, Macromolecules, 2, 341 (1969).

7. A. Stroeks and E. Nies, Macromolecules, 23, 4092 (1990).

8. E. Nies and H. Xie, Macromolecules, 26, 1683 (1993).

9. H. Xie and E. Nies, Macromolecules, 26, 1689 (1993).

10. R. J. Roe and D. Rigby, Adv. Polym. Sci., 82, 105 (1986).

11. P. Zoller R. K. Jain, and R. Simha, J. Polym. Sci. Part B: Polym. Phys., 24, 687 (1986).

12. G. R. Strobl, J. T. Bendler, R. P. Kambour, and A. R. Shultz, Macromolecules, 19, 2683 (1986).

13. Experimental data for $\mathrm{p}(\mathrm{pBrS})$ and $\mathrm{p}(\mathrm{S}-\mathrm{co}-\mathrm{p}-\mathrm{BrS})$, to be published.

APPENDIX A: List of Symbols for the Theory of Random Copolymer/Homopolymer Blends

$\varphi_{\mathrm{A}}\left(\varphi_{\mathrm{B}}\right)$ the segment fraction of component $\mathrm{A}(\mathrm{B})$

$\langle s\rangle \quad$ the average number of segment per molecule

$y \quad$ occupied site fraction

$z \quad$ the coordinate number of the lattice

$u_{1} \quad$ contact site fraction of component $\mathrm{A}_{1}$ in the copolymer

$u_{2} \quad$ contact site fraction of component $\mathrm{A}_{2}$ in the copolymer

$v \quad$ contact site fraction of homopolymer B

$w \quad$ contact site fraction of holes

$\left\langle c_{\mathrm{s}}\right\rangle \quad$ the average flexibility parameter

$\left\langle v^{*}\right\rangle$ the average hard core volume of each segment in the blend

$\tilde{\omega} \quad$ reduced cell volume

$\widetilde{T} \quad$ reduced temperature

$\gamma \quad$ an auxiliary parameter, defined by $\gamma=2 / z$

$\langle\alpha\rangle \quad$ an auxiliary parameter, defined by $\langle\alpha\rangle=\gamma(1-1 /$ $\langle s\rangle)$

$\beta \quad$ an auxiliary parameter, defined by $\beta=2^{-1 / 6} \tilde{\omega}^{-1 / 3}$

APPENDIX B: The Equation of State for Random Copolymer/Homopolymer Blends

The equation of state of a binary mixture is given by

$$
P=-\left.\frac{\partial A}{\partial V}\right|_{N, Y, y, \varphi_{A}}
$$

For random copolymer/homopolymer blends, we have

$$
\begin{aligned}
\frac{\tilde{P} \tilde{V}}{\tilde{T}}= & \frac{1}{1-\beta\left(1-\frac{\bar{x}}{q}\right)}+\frac{2}{\tilde{T}}\left(1-\frac{\bar{x}}{q}\right)\left(A \tilde{\omega}^{-4}-B \tilde{\omega}^{-2}\right) \\
& +\sum_{j=1}^{6}\left(m_{2 j}+m_{3 j}+\frac{1}{2 \tilde{T}} m_{4 j}\right) V \frac{\partial \bar{x}_{j}}{\partial V}
\end{aligned}
$$

In eq $\mathrm{B} 2$

$$
m_{2 j}=\frac{z(1-\langle\alpha\rangle)}{q\left\langle c_{\mathrm{s}}\right\rangle} \ln \eta_{j} \quad(j=1,2,4)
$$

$$
\begin{aligned}
& m_{2 j}= \frac{z(1-\langle\alpha\rangle)}{2 q\left\langle c_{\mathrm{s}}\right\rangle}\left(2 \ln \eta_{j}-\ln f_{\mathrm{e}}\right) \quad(j=3,5,6) \\
& m_{3 j}= {\left[1-\beta\left(1-\frac{\bar{x}}{q}\right)\right]^{-1}\left[-\beta\left(1-\frac{\bar{x}}{q}\right) \frac{\partial \ln \left\langle v^{*}\right\rangle}{\partial \bar{x}_{j}}\right] } \\
& m_{3 j}= {\left[1-\beta\left(1-\frac{x}{q}\right)\right]^{-1}\left[\frac{3 \beta}{q}-\beta\left(1-\frac{\bar{x}}{q}\right) \frac{\partial \ln \left\langle v^{*}\right\rangle}{\partial \bar{x}_{j}}\right] } \\
& m_{4 j}=-\left\{\left(1-\frac{\bar{x}}{q}\right)\left[\left(A \tilde{\omega}^{-4}-2 B \tilde{\omega}^{-2}\right) \frac{\partial \ln \left\langle\varepsilon^{*}\right\rangle}{\partial \bar{x}_{j}}\right]\right. \\
&\left.+4\left(1-\frac{\bar{x}}{q}\right)\left(A \tilde{\omega}^{-4}-B \tilde{\omega}^{-2}\right) \frac{\partial \ln \left\langle v^{*}\right\rangle}{\partial \bar{x}_{j}}\right\} \\
& m_{4 j}=-\left\{\left[\left(1-\frac{\bar{x}}{q}\right) \frac{\partial \ln \left\langle\varepsilon^{*}\right\rangle}{\partial \bar{x}_{j}}-\frac{1}{q}\right]\left(A \tilde{\omega}^{-4}-2 B \tilde{\omega}^{-2}\right)\right. \\
&\left.+4\left(1-\frac{\bar{x}}{q}\right)\left(A \tilde{\omega}^{-4}-B \tilde{\omega}^{-2}\right) \frac{\partial \ln \left\langle v^{*}\right\rangle}{\partial \bar{x}_{j}}\right\} \\
&(j=3,5,6)
\end{aligned}
$$

where

$$
\begin{aligned}
\eta_{1}= & \exp \left\{\left[2 \varepsilon_{\mathrm{A}_{1} \mathrm{~A}_{1}}^{*}\left(A \tilde{\omega}_{12}^{-4}-2 B \tilde{\omega}_{12}^{-2}\right)\right.\right. \\
& -\varepsilon_{\mathrm{A}_{1} \mathrm{~A}_{1}}^{*}\left(A \tilde{\omega}_{1}^{-4}-2 B \tilde{\omega}_{1}^{-2}\right) \\
& \left.\left.-\varepsilon_{\mathrm{A}_{2} \mathrm{~A}_{2}}^{*}\left(A \tilde{\omega}_{2}^{-4}-2 B \tilde{\omega}_{2}^{-2}\right)\right] / 2 k T\right\} \\
\eta_{2}= & \exp \left\{\left[2 \varepsilon_{\mathrm{A}_{1} \mathrm{~B}}^{*}\left(A \tilde{\omega}_{1 \mathrm{~B}}^{-4}-2 B \tilde{\omega}_{1 \mathrm{~B}}^{-2}\right)\right.\right. \\
& -\varepsilon_{\mathrm{A}_{1} \mathrm{~A}_{1}}^{*}\left(A \tilde{\omega}_{1}^{-4}-2 B \tilde{\omega}_{1}^{-2}\right) \\
& \left.\left.-\varepsilon_{\mathrm{BB}}^{*}\left(A \tilde{\omega}_{\mathrm{B}}^{-4}-2 B \tilde{\omega}_{\mathrm{B}}^{-2}\right)\right] / 2 k T\right\} \\
\eta_{3}= & \exp \left\{-\varepsilon_{\mathrm{A}_{1} \mathrm{~A}_{1}}^{*}\left(A \tilde{\omega}_{1}^{-4}-2 B \tilde{\omega}_{1}^{-2}\right) / 2 k T\right\} \\
\eta_{4}= & \exp \left\{\left[2 \varepsilon_{A_{2} \mathrm{~B}}^{*}\left(A \tilde{\omega}_{2 \mathrm{~B}}^{-4}-2 B \tilde{\omega}_{2 \mathrm{~B}}^{-2}\right)\right.\right. \\
& -\varepsilon_{\mathrm{A}_{2} \mathrm{~A}_{2}}^{*}\left(A \tilde{\omega}_{2}^{-4}-2 B \tilde{\omega}_{2}^{-2}\right) \\
& \left.\left.-\varepsilon_{\mathrm{BB}}^{*}\left(A \tilde{\omega}_{\mathrm{B}}^{-4}-2 B \tilde{\omega}_{\mathrm{B}}^{-2}\right)\right] / 2 k T\right\} \\
\eta_{5}= & \exp \left\{-\varepsilon_{\mathrm{A}_{2} \mathrm{~A}_{2}}^{*}\left(A \tilde{\omega}_{2}^{-4}-2 B \tilde{\omega}_{2}^{-2}\right) / 2 k T\right\} \\
\eta_{6}= & \exp \left\{-\varepsilon_{\mathrm{BB}}^{*}\left(A \tilde{\omega}_{\mathrm{BB}}^{-4}-2 B \tilde{\omega}_{\mathrm{BB}}^{-2}\right) / 2 k T\right\} \\
\bar{x}= & \bar{x}_{3}+\bar{x}_{5}+\bar{x}_{6}
\end{aligned}
$$

and

$$
f_{\mathrm{e}}=\exp \left\{\frac{6\left\langle c_{\mathrm{s}}\right\rangle}{z(1-\langle\alpha\rangle)} \frac{\beta}{\left[1-\beta\left(1-\frac{\bar{x}}{q}\right)\right]}\right\}
$$

(The different indices in the above expressions indicate that the cell volume is reduced with different hard core volumes.) 